\title{
BeCAS ESCOLARES Y SU IMPACTO EN LA PERCEPCIÓN DEL PROMEDIO ESCOLAR: EVIDENCIA PARA LA Ciudad DE MÉxico
}

\author{
Armando Sánchez Vargas, Verónica Villarespe \\ y Anadeli Naranjo ${ }^{a}$
}

Fecha de recepción: 20 de noviembre de 2019. Fecha de aceptación: 15 de febrero de 2020.

http://doi.org/10.22201/iiec.20078951e.2020.201.69536

Resumen. El presente artículo muestra los resultados de impacto de un Programa de Becas Escolares de la Ciudad de México sobre la percepción que las familias tienen del promedio escolar de sus hijas e hijos. El estudio emplea una nueva base de datos recolectada para la evaluación de dicho programa de transferencias monetarias condicionadas, que contiene información para una muestra aleatoria sobre un grupo de tratamiento y un grupo de control. Por medio de modelos de regresión lineal y probit ordenado, se encontró que la participación en el Programa de Becas Escolares aumentó $18.1 \%$ la percepción de promedios altos y redujo 11.3 y $6.7 \%$ la percepción de promedios de rango medio y bajo, respectivamente.

Palabras clave: educación; política educativa; Programa de Becas Escolares; percepción de promedio escolar; modelo econométrico probit.

Clasificación JEL: C23; C33; I21; I22; J13.

\section{SCHOLARSHIPS AND THEIR IMPACT ON THE PERCEPTION OF GRADE AVERAGES: EVIDENCE FROM MEXICO City}

\begin{abstract}
This paper presents the results of the impact that a Scholarship Program in Mexico City has on perceptions that families have of their children's grade averages. The study uses a new database collected for the purpose of evaluating this conditional cash transfer program, which contains information for a random sample of a treatment group and control group. Through linear and ordered probit regression models, it was found that participation in the Scholarship Program increased the perception of high grade averages by $18.1 \%$, and reduced 11.3 and $6.7 \%$ the perception of mid and low range grade averages, respectively.

Key Words: education; education policy; Scholarship Programs; perception of grade averages; probit econometric model.

\footnotetext{
${ }^{a}$ Universidad Nacional Autónoma de México (UNAM), Instituto de Investigaciones Económicas, México.Correos electrónicos: asvargas@unam.mx; reyesvv@unam.mx y anadeli_28@hotmail.com, respectivamente.
} 


\section{INTRODUCCIÓN ${ }^{1}$}

Un hecho ampliamente reconocido en la literatura económica es que la inversión en capital humano juega un papel determinante en el crecimiento económico de un país. En particular, la literatura que analiza los programas de políticas públicas de Becas Escolares por lo general se basa en un marco teórico de formación de capital humano.

Asimismo, existe también evidencia empírica que constituye el marco de referencia para analizar este tipo de políticas ${ }^{2}$ que sugiere que la inversión en la cantidad y calidad de la educación que reciben los individuos depende del retorno esperado que perciben los padres (Woessmann y Hanushek, 2012; Glewwe, 2002). En consecuencia, en los países en desarrollo el reto es la aplicación de políticas públicas que hagan más rentable la inversión en educación para las familias $y$, de este modo, se obtengan incentivos para que niñas y niños permanezcan más años en la escuela.

Bajo este contexto, una de las estrategias es la aplicación de programas de transferencias monetarias que están condicionadas a una mayor participación escolar. Estas políticas públicas podrían tener impactos positivos en la rentabilidad que perciben las familias, debido a que generan un aumento inmediato en el ingreso disponible del hogar del menor beneficiado, pero también propician un cambio en la percepción de los hogares sobre la cantidad y calidad del capital humano de quienes reciben la beca. El resultado podría llevar a un aumento en las expectativas del retorno.

En 2001, se implementó el Programa de Becas Escolares para niñas y niños en condición de vulnerabilidad social de la Ciudad de México ${ }^{3}$ (Programa de Becas Escolares de aquí en adelante), cuyo objetivo es aumentar la participación y permanencia escolar de niñas y niños en situación de vulnerabilidad que se encuentran en edad de cursar la educación básica. El programa consiste en otorgar una transferencia monetaria mensual de MXN\$800 a las familias participantes, que está condicionada a la inscripción de los menores en el ciclo escolar correspondiente.

La lógica de esta beca es contribuir a la permanencia escolar de los beneficiarios y a través de ella incidir sobre su desempeño escolar. Lo anterior es

1 Investigación realizada gracias al Programa UNAM-PAPIIT IN302419, PAPIME PE310919 y al Consejo de Evaluación del Desarrollo Social de la Ciudad de México (EVALUA-CDMX).

2 Lo autores agradecen a un árbitro anónimo el comentario sobre la posibilidad de que existen otros marcos de referencia para analizar el tema de educación y que las experiencias que se dan en otros países podrían ser un referente para el análisis del tema.

3 También llamado "Más becas, mejor educación". 
muy posible, ya que los recursos disponibles de las familias se ven aumentados para invertir en educación, salud y alimentación. De tal forma que existe una mayor motivación por parte de los padres de familia o tutores para que sus hijos permanezcan en la escuela.

El principal objetivo de este artículo es determinar el impacto del Programa de Becas Escolares de la Ciudad de México en la percepción de los padres sobre el rendimiento académico de sus hijas e hijos. Para ello, se hizo uso de una nueva base de datos resultante de una encuesta representativa que comprende todas las alcaldías de la Ciudad de México y que fue levantada específicamente para la evaluación del impacto del programa.

Con la finalidad de estimar dichos impactos se utilizó un modelo teórico de los determinantes de las calificaciones escolares a nivel hogar, en donde se contempla la incidencia de un programa de política pública, así como el modelo de regresión múltiple y el modelo probit ordenado, este último permitió tomar en cuenta la naturaleza categórica de la variable dependiente. Con base en dicho modelo de probit ordenado, se estimaron los efectos marginales para las diferentes categorías de respuesta.

Los resultados empíricos del modelo sugieren que existen impactos positivos y significativos del Programa de Becas Escolares de la Ciudad de México en la percepción que tienen las familias sobre las calificaciones de sus hijas e hijos. De hecho, los padres de los participantes en el programa tienen una percepción de que las calificaciones que obtienen sus hijas e hijos en el ciclo escolar son más altas.

Específicamente, la probabilidad de que los padres perciban una calificación alta (entre nueve y diez) aumenta $18.1 \%$ para los beneficiarios en relación con la percepción de los padres del grupo de control. Al mismo tiempo, la probabilidad de que los padres perciban una calificación menor (de nueve) disminuye de manera significativa en el grupo tratado (la percepción de las calificaciones entre ocho y nueve se reduce $11.3 \%$ y de calificaciones entre siete y ocho en $6.7 \%$ ) en comparación con los no tratados.

Este artículo se compone de seis secciones, incluyendo esta introducción. La segunda explica la operación del Programa de Becas Escolares y resume los antecedentes en la evaluación de programas de transferencias monetarias y su incidencia sobre los logros escolares a modo de comparación para diversos países. La tercera sección presenta la estadística descriptiva de las variables colectadas en la encuesta. La cuarta expone el marco teórico y la estrategia de estimación. La quinta sección analiza los resultados de impacto cuantitativo en la percepción. Y finalmente, se discuten de manera breve las conclusiones en la sexta sección. 


\section{EL PROGRAMA DE BECAS ESCOLARES Y LA EVIDENCIA EMPÍRICA}

El Programa de Becas Escolares de la Ciudad de México fue aplicado por primera vez en 2001, su finalidad es contribuir a que nińas y niños en situación de vulnerabilidad social ${ }^{4}$ permanezcan más tiempo en la escuela y concluyan sus estudios de nivel básico. La entidad encargada para la operación del programa es el Sistema para el Desarrollo Integral de la Familia de la Ciudad de México (DIf-CDMx). De acuerdo con cifras del Consejo Nacional de Evaluación de la Política de Desarrollo Social (coneval), en 2015 existían 246 473 niñas y niños vulnerables por carencias sociales (Gaceta Oficial Distrito Federal, 2015-2018).

En 2015, el programa atendió a 25500 estudiantes de entre seis y catorce años de edad, inscritos en escuelas públicas de nivel primaria o secundaria, a los que se les otorgó el apoyo condicionado a la permanencia escolar, y que consistió en una beca de MXN\$800 mensuales por un periodo máximo de tres años. El presupuesto destinado al programa en dicho ejercicio presupuestal ascendió a MXN\$24800 000. Otro eje que forma parte de esta intervención es la participación de los beneficiarios en actividades culturales y recreativas (Gaceta Oficial Distrito Federal, 2015-2018).

Los programas de transferencias monetarias condicionadas a la inscripción escolar de niñas y niños han sido implementados en distintos países en desarrollo en décadas recientes (Fiszbein et al., 2009; Inter-American Development Bank, 2017; Stampini y Tornarolli, 2012). Existe evidencia de que este tipo de programas han tenido un importante crecimiento en la región de América Latina y el Caribe; asimismo, se ha generado cierto consenso sobre la existencia de una relación causal entre los programas de transferencias monetarias y los indicadores escolares de permanencia, tales como inscripción, asistencia y grados completados (Glewwe y Kassouf, 2012; Brauw et al., 2015; Behrman et al., 2009).

Sin embargo, para que este tipo de programas tengan un impacto más apropiado se requiere que generen mayores beneficios en la adquisición efectiva de habilidades y conocimientos necesarios para enfrentar los requerimientos laborales futuros (Woessmann y Hanushek, 2012). Una forma tradicional para evaluar la adquisición de competencias en los estudiantes es mediante

4 De acuerdo con las reglas de operación del programa, son consideradas personas en situación de vulnerabilidad quienes cuentan con un ingreso mensual superior a la línea de bienestar, pero que presentan alguna carencia social (educación, salud, alimentación, calidad de la vivienda, seguridad social, servicios básicos de la vivienda). 
la permanencia escolar y la asignación de calificaciones. Algunos programas de transferencias monetarias instrumentados en países en desarrollo muestran una incidencia positiva sobre los resultados de indicadores de desempeño escolar. Este artículo se enfoca en el análisis de la percepción del promedio escolar por parte de los padres y las madres de familia, así como su variación por medio de la participación en un programa público de Becas Escolares de la Ciudad de México.

$\mathrm{Al}$ respecto, Stampini et al. (2018) estudian los impactos del Programa de Avances a través de la Salud y la Educación (PATH), que es un programa de transferencias monetarias condicionadas a la asistencia escolar de educación básica en Jamaica. Este estudio generó resultados sobre dos tipos de logros escolares: $a$ ) las calificaciones académicas; y b) la colocación en escuelas secundarias. Sus hallazgos sugieren que existe una ganancia en los puntajes escolares de estudiantes hombres, atribuible a la recepción de la transferencia monetaria. Del mismo modo, la asignación en escuelas secundarias de mejor ranking fue también positiva para los niños beneficiarios del programa en comparación con los no beneficiarios. Sin embargo, los autores no encontraron evidencia de efectos significativos en los resultados escolares de las niñas.

Otro programa de transferencias monetarias condicionado a la permanencia escolar es el evaluado por Simóes y Sabates (2014), el cual es asignado a familias pobres en Brasil. Los hallazgos de su investigación, basados tanto en un modelo de sección cruzada como de panel con efectos fijos, arrojan un impacto positivo sobre las calificaciones de pruebas estandarizadas de los estudiantes que reciben el beneficio, el cual además es sensible al tiempo de exposición al programa.

Garcia y Hill (2010) estudian los efectos del programa colombiano de transferencias condicionadas ("Familias en Acción") sobre las calificaciones escolares de niños y adolescentes. Mediante la técnica de Propensity Score Matching, los autores encuentran un efecto positivo del programa sobre los puntajes de pruebas académicas. Aunque esta relación es cierta para los estudiantes de entre siete y doce ańos de zonas rurales, en las zonas urbanas no presenta efectos significativos. Por lo tanto, el efecto es negativo entre adolescentes de trece a diecisiete ańos.

Por otro lado, existen otros factores tanto observables como no observables que determinan los resultados de aprendizaje de niñas y niños. Algunos estudios muestran que las características individuales juegan un papel importante. Específicamente, la evidencia señala que la función que tradicionalmente algunas familias les asignan a las hijas continúa siendo un determinante de los resultados de las calificaciones de los estudiantes. 


\section{DATOS}

Los datos provienen de la encuesta a hogares diseñada específicamente para la evaluación de impacto del Programa de Becas Escolares para niñas y niños en condición de vulnerabilidad social de la Ciudad de México. Esta encuesta es la primera que se lleva a cabo para el análisis de los efectos del programa, por lo cual, representa la línea de base. Las encuestas fueron levantadas durante el mes de enero de 2016, su diseńo sigue un enfoque comparativo entre grupos de tratamiento y control, y tiene una representatividad a nivel alcaldía para toda la Ciudad de México. Dicha encuesta proporciona información sobre la situación de estudiantes de primaria y secundaria en el ciclo escolar 2014-2015, así como de un conjunto amplio de variables de sus familias. Es importante destacar que el programa no tuvo modificaciones considerables en su diseño y operación desde su creación en 2001 hasta el momento de la evaluación en 2016 (Gaceta Oficial Distrito Federal, 2016).

Todos los estudiantes de primaria o secundaria pública de la Ciudad de México fueron considerados elegibles para el estudio si presentaban alguna de las siguientes condiciones: a) ser beneficiario del Programa de Becas Escolares durante el ciclo escolar 2014-2015, y b) estar registrado en la lista de espera para acceder al programa en el mismo periodo. Por ello, la población de estudio fueron 25500 beneficiarios (grupo de tratamiento) y 1516 estudiantes en lista de espera (grupo de control). De dichas poblaciones, se seleccionó una muestra aleatoria con estratificación por alcaldía de 201 niñas y niños, donde $104(51.7 \%)$ estudiantes pertenecieron al grupo de tratamiento y $97(48.3 \%)$ al de control.

La información de los estudiantes sobre su condición de participación en el Programa de Becas Escolares se obtuvo directamente a partir de los registros administrativos del programa. Sin embargo, para corroborar su estatus fue implementado un filtro al inicio del cuestionario, de este modo se aseguró la correcta asignación de los alumnos en cada grupo, ya fuera como beneficiarios o de lista de espera. Con este procedimiento se descartó a quienes no cumplieron las condiciones para estar en uno u otro grupo, en el ciclo escolar evaluado, a pesar de estar listados en los padrones.

En la encuesta se recolectó información de la percepción de madres, padres y tutores ${ }^{5}$ sobre las calificaciones generales de sus hijas e hijos durante el ciclo

5 Para efectos de la encuesta, el tutor hace referencia a la persona responsable de los asuntos escolares de niñas y niños. 
escolar 2014-2015, con esto se obtuvo el indicador de resultado escolar. ${ }^{6}$ Este indicador contó con tres categorías de respuesta: la primera de ellas se refiere a la percepción de calificaciones entre nueve y diez; la segunda a la de entre ocho y nueve, y la tercera a calificaciones entre siete y ocho. Los alumnos fueron considerados para el estudio únicamente si los tutores proporcionaron información de su promedio general. ${ }^{7}$ En esa medida, el indicador presentó valores para un total de 182 de los 201 estudiantes seleccionados. ${ }^{8}$

Una primera observación de los datos (véase gráfica 1) muestra que, en opinión de las madres, padres y tutores, los estudiantes tanto beneficiarios como no beneficiarios tienen porcentajes muy parecidos en cuanto a promedios de entre nueve y diez ( 44.2 y $44.8 \%$ cada grupo, respectivamente), que podrían ser categorizados como las mejores calificaciones. Mientras que el porcentaje de estudiantes con promedios intermedios de entre ocho y nueve es superior en el grupo de control (42.7\%) que en el de tratamiento (36.1\%). Por su parte, el porcentaje de estudiantes con promedios de menor rango (entre siete y ocho) es mayor entre el grupo de beneficiarios (19.8\%) en relación con el de no beneficiarios (12.5\%).

Si la asignación en el programa se realizara de forma aleatoria, estos datos podrían señalar la estimación precisa entre la participación en el programa y el resultado de la percepción de calificaciones; sin embargo, debido a que la asignación no sigue esta forma, los resultados de diferencias simples no pueden interpretarse aún en términos causales, pues no contemplan el impacto de otras características tanto observables como no observables sobre el resultado del indicador.

6 Existe un intenso debate pedagógico intrínseco y extrínseco a la calificación. Del intrínseco no es posible determinar si el siete de una escuela es idéntico al siete en otra escuela o de un maestro a otro maestro. Respecto al extrínseco se cuestiona si una calificación refleja el aprendizaje, sobre todo cuando es un proceso complejo y la calificación sólo refleja evidencias indirectas. Los autores agradecen a uno de los árbitros de este artículo por este comentario.

7 Los estudiantes cuyos tutores no pudieron brindar información de la percepción de promedio general o decidieron no dar este dato fueron descartados del estudio.

8 Las calificaciones de primaria y secundaria se expresan en una escala de cinco a diez Los alumnos de primero y segundo grado de primaria son evaluados por una escala de seis a diez y pueden aprobar el grado escolar sólo con asistir (Diario Oficial de la Federación, 2019), sin embargo, es posible evaluar el indicador de percepción de promedio general porque dicha escala se encuentra en el rango de interés para el indicador.

La distribución de la muestra por nivel de inscripción refleja los porcentajes de estudiantes en cada nivel dentro de la población objetivo. Así, 29.4\% fueron estudiantes de secundaria y $70.6 \%$ de primaria. 
Gráfica 1. Percepción del promedio en el ciclo escolar 2014-2015 por grupos de tratamiento y control

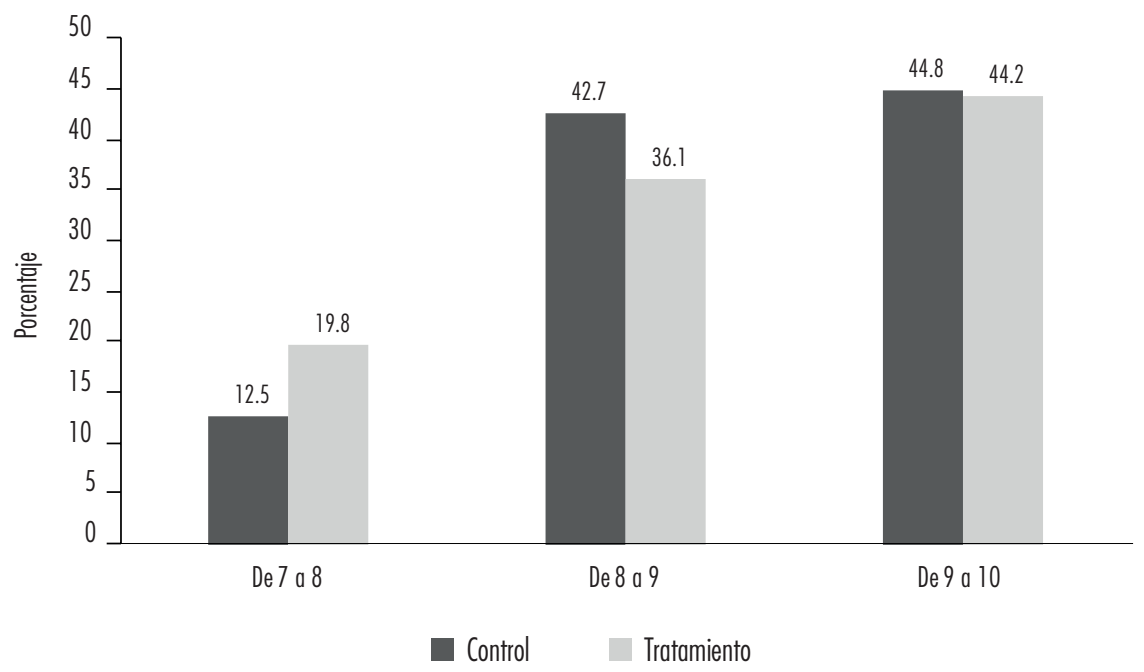

Fuente: elaboración propia con datos de la Encuesta de Evaluación de Impacto del Programa de Becas Escolares de la Ciudad de México (2016).

Para controlar todas las características que podrían incidir en el resultado final de la percepción de calificaciones, se incorporaron los grupos de regresores que, de acuerdo con la evidencia empírica (Glewwe y Lambert, 2010; Glewwe y Kremer, 2006), conforman una función de producción de los logros escolares. El cuadro 1 muestra el resumen estadístico de las principales variables de cada grupo: características individuales, familiares y de la localidad, adicionalmente se controla por la participación en el programa de política pública.

La primera, segunda y tercera columna de cada cuadro presentan los valores medios de cada variable, así como su desviación estándar, esto para el total de la muestra, el grupo de control y el de tratamiento, respectivamente. La cuarta columna muestra el resultado de diferencia de medias simple de cada característica entre tratados y no tratados. En la quinta columna se realiza la misma prueba, pero contemplando adicionalmente los efectos de la ubicación geográfica.

Los datos del cuadro 1 señalan que la muestra de estudiantes está compuesta por $50.7 \%$ de niñas y $49.3 \%$ de niños. La distribución de dichos estudiantes, de acuerdo con su nivel de inscripción, es de $29.4 \%$ en secundaria y 
Cuadro 1. Características de los estudiantes

\begin{tabular}{lccccc}
\hline Característica & Total & $\begin{array}{c}\text { Estudiantes de } \\
\text { tratamiento }\end{array}$ & $\begin{array}{c}\text { Estudiantes de } \\
\text { control }\end{array}$ & P-value & $\begin{array}{c}\text { P-value con efectos } \\
\text { fijos por alcaldía }\end{array}$ \\
\hline Género: femenino & 50.7 & 48.1 & 53.6 & 0.436 & 0.393 \\
Nivel: secundaria & $(0.50)$ & $(0.00)$ & $(0.00)$ & & 0.084 \\
Relación con & 29.4 & 34.6 & 23.7 & 0.091 & 0.528 \\
compañeros & $(0.46)$ & $(0.47)$ & $(0.42)$ & & \\
Comportamiento & 83.8 & 82.5 & 85.3 & 0.603 & 0.291 \\
en la escuela & $(0.37)$ & $(0.38)$ & $(0.35)$ & & \\
Horas de estudio & $(0.27)$ & $(0.29)$ & $(0.24)$ & & 0.413 \\
& 1.24 & 1.21 & 1.27 & 0.546 & \\
Hijo del jefe & $(0.79)$ & $(0.81)$ & $(0.76)$ & & 0.440 \\
del hogar & 90.05 & 91.3 & 88.6 & 0.527 & \\
\hline
\end{tabular}

Fuente: elaboración propia con datos de la Encuesta de Evaluación de Impacto del Programa de Becas Escolares de la Ciudad de México (2016).

$70.6 \%$ en primaria $;{ }^{9} 83.8 \%$ de los tutores consideran que sus hijos tienen una buena relación con sus compañeros y $91.9 \%$ señalan que también presentan un buen comportamiento en la escuela. En promedio, los alumnos dedican 1.2 horas a estudiar diariamente, adicional al tiempo que dedican a realizar sus tareas; $90.0 \%$ de los estudiantes tienen una relación de hijo del jefe del hogar.

Los antecedentes familiares se presentan en el cuadro 2. Los datos muestran que $91.5 \%$ de los tutores es del género femenino y en $81.9 \%$ de los casos la madre es la responsable directa de las actividades escolares de los estudiantes; $15.4 \%$ de los tutores tienen como nivel de estudios máximo la primaria completa y $8.7 \%$ tienen secundaria incompleta. Los tutores reportan estar empleados $64.7 \%$ de las veces. En términos de acompañamiento escolar, $86.6 \%$ de los tutores mencionan revisar las tareas de los niños con alta regularidad, el resto lo hace con poca o nula frecuencia. gundo grado, donde la escala es de seis a diez (Diario Oficial de la Federación, 2019). 
Armando Sánchez, Verónica Villarespe y Anadeli Naranjo

Cuadro 2. Características de los tutores

\begin{tabular}{lccccc}
\hline Característica & Total & $\begin{array}{c}\text { Estudiantes de } \\
\text { tratamiento }\end{array}$ & $\begin{array}{c}\text { Estudiantes de } \\
\text { control }\end{array}$ & P-value & $\begin{array}{c}\text { P-value con efectos } \\
\text { fijos por alcaldía }\end{array}$ \\
\hline Género: femenino & 91.54 & 95.1 & 87.6 & 0.055 & 0.073 \\
& $(0.28)$ & $(0.21)$ & $(0.33)$ & & 0.220 \\
La madre es el tutor & 81.91 & 85.2 & 78.3 & 0.205 & 0.430 \\
& $(0.39)$ & $(0.35)$ & $(0.41)$ & & \\
Escolaridad: & 15.38 & 12.7 & 18.3 & 0.287 & 0.297 \\
primaria completa & $(0.36)$ & $(0.33)$ & $(0.38)$ & & \\
Escolaridad: & 8.72 & 8.8 & 4.3 & 0.208 & 0.511 \\
secundaria incompleta & $(0.28)$ & $(0.28)$ & $(0.20)$ & & \\
Empleo & 64.68 & 68.27 & 60.8 & 0.503 & 0.986 \\
& $(0.48)$ & $(1.53)$ & $(1.62)$ & & \\
Frecuencia de ayuda & 86.57 & 86.50 & 86.50 & 0.990 & \\
con las tareas & $(0.34)$ & $(0.34)$ & $(0.34)$ & & \\
\hline
\end{tabular}

Fuente: elaboración propia con datos de la Encuesta de Evaluación de Impacto del Programa de Becas Escolares de la Ciudad de México (2016).

El cuadro 3 presenta las características de los hogares y de la comunidad. El $61.7 \%$ de estos hogares disponen de teléfono en casa; en cambio, apenas $40.8 \%$ cuenta con Internet en su vivienda. El gasto mensual promedio que los hogares vulnerables destinan a educación es de $\mathrm{MXN} \$ 318.3$. Por su parte, el gasto dedicado a salud es tan sólo de $\mathrm{MXN} \$ 118.4$ al mes, mientras que el gasto dedicado a vivienda es de MXN\$468.8. En cuanto a las condiciones de la comunidad, un factor que podría ser relevante es el tiempo de desplazamiento que los estudiantes realizan para ir de su casa a la escuela. Los integrantes de la muestra tardan en promedio 31.5 minutos en este desplazamiento.

Las pruebas de diferencia de medias en las variables, tanto en su forma simple como controlando los efectos fijos de la ubicación geográfica, no señalan discrepancias estadísticamente significativas en ninguna de los grupos. Lo anterior podría indicar que los dos grupos son altamente comparables. 
Cuadro 3. Características de los hogares y la comunidad

\begin{tabular}{lccccc}
\hline Característica & Total & $\begin{array}{c}\text { Estudiantes de } \\
\text { tratamiento }\end{array}$ & $\begin{array}{c}\text { Estudiantes de } \\
\text { control }\end{array}$ & $\begin{array}{c}\text { P-value } \\
\text { P-value con efectos } \\
\text { fijos por alcaldía }\end{array}$ \\
\hline Hogar & 61.69 & 64.4 & 58.8 & 0.412 & 0.253 \\
\hline Teléfono & $(0.49)$ & $(0.48)$ & $(0.49)$ & & \\
Internet & 40.80 & 39.4 & 42.3 & 0.684 & 0.557 \\
Gasto en educación & $(0.49)$ & $(0.49)$ & $(0.49)$ & & \\
& 318.3 & 325.3 & 311.2 & 0.662 & 0.766 \\
Gasto en salud & $(222.6)$ & $(209.4)$ & $(236.2)$ & & 0.647 \\
& 118.4 & 123.8 & 112.4 & 0.649 & \\
Gasto en vivienda & $(172.0)$ & $(180.6)$ & $(162.8)$ & & 0.757 \\
\hline Comunidad & 468.8 & 461.0 & 477.2 & 0.819 & \\
\hline $\begin{array}{l}\text { Tiempo de } \\
\text { desplazamiento } \\
\text { a la escuela }\end{array}$ & $(499.0)$ & $(500.2)$ & $(500.1)$ & & \\
\hline
\end{tabular}

Fuente: elaboración propia con datos de la Encuesta de Evaluación de Impacto del Programa de Becas Escolares de la Ciudad de México (2016).

\section{MARCO DE REFERENCIA}

El proceso de adquisición de logros escolares en estudiantes puede analizarse desde el enfoque de una función de producción. Por ello, siguiendo el marco teórico desarrollado por Glewwe y Lambert (2010) y Glewwe y Kremer (2006), se empleó esta metodología para evaluar el impacto de esta política pública destinada a apoyar los logros escolares de niñas y niños vulnerables en la Ciudad de México. De manera general, la adquisición de los logros escolares puede estar determinada por variables asociadas al estudiante, características de sus hogares y particularidades de sus escuelas, tal como se representa en la ecuación 1 . 


$$
L=l(A E, N, H, I, E)
$$

En esta primera ecuación, $L$ son los logros escolares de niñas y niños. $A E$ son los años de escolaridad. $N$ es el conjunto de características de los estudiantes y comprende también variables no observables como la habilidad innata. $H$ son todos los antecedentes de los hogares que inciden sobre los logros escolares. I representa las inversiones que hacen las familias en la educación de niñas y niños, donde dichas entradas pueden ser monetarias o de tiempo de acompañamiento. Finalmente, $E$ se refiere a todos los factores relacionados con la calidad de las escuelas y profesores que podrían fomentar cambios en los resultados escolares.

En esta ecuación, los impactos de cada característica pueden interpretarse manteniendo el resto de los factores constantes. Sin embargo, la forma en que algunas variables afectan a los logros escolares no es únicamente directa como la representada en la ecuación 1; sino que cambios en las variables de calidad de las escuelas y profesores $(E)$ podrían originar modificaciones sobre las decisiones de los hogares; es decir, aumentar o disminuir las inversiones que las familias realizan en educación, así como los años de estudio que eligen para sus hijos.

Por lo tanto, el efecto de la variación en las condiciones escolares podría no estar capturado por completo. Para obtener el impacto total de dichos cambios, puede plantearse una forma reducida de la función de producción de habilidades escolares. Para ello, se expresa primero tanto los años de escolaridad como las inversiones que las familias destinan a educación se encuentran en función de los cambios en $E$ y en otras variables. Esta relación se representa por medio de las ecuaciones 2 y 3 .

$$
\begin{aligned}
& A E=a e(N, H, P, E) \\
& I=i(N, H, P, E)
\end{aligned}
$$

Tanto en 2 como en 3, $P$ representa a los precios que afectan las decisiones de educación de los hogares. Entonces, sustituyendo las ecuaciones 2 y 3 en 1 , se obtiene la siguiente forma reducida de la función de logros escolares:

$$
L=l(N, H, P, E)
$$

Si bien la ecuación 4 contempla ya los efectos indirectos que generan los cambios en las variables sobre el resultado de logros escolares, aún es necesario 
valorar el impacto de las políticas públicas. Las políticas que el gobierno implementa para apoyar los logros educativos de los estudiantes pueden incidir sobre las variables de calidad de las escuelas, así como sobre los precios de educación que perciben los hogares, por lo cual se presentan las ecuaciones 5 y 6 que representan la relación descrita.

$$
\begin{aligned}
& E=e(C, P P) \\
& P=p(C, P P)
\end{aligned}
$$

Las ecuaciones 5 y 6 contemplan la interacción de las políticas públicas con las características de las comunidades donde habitan los estudiantes, así como su incidencia sobre la calidad de las escuelas y los precios de la educación. Así, podemos sustituir 5 y 6 en 2 y 4 para obtener la forma reducida de la ecuación de logros escolares que contemple en su totalidad el impacto causal de los diferentes factores sobre el indicador de logros escolares.

$$
\begin{aligned}
& A E=a e(N, H, C, P P) \\
& L=l(N, H, C, P P)
\end{aligned}
$$

La ecuación 8 es de sumo interés para la evaluación de un programa de política pública de Becas Escolares sobre la percepción de calificaciones escolares en estudiantes de la Ciudad de México. Finalmente, la ecuación anterior puede ser expresada en términos de una regresión como:

$$
\begin{aligned}
L= & \beta_{0}+\beta_{1} A E+\beta_{N 1} N_{1}+\beta_{N 2} N_{2}+\cdots+\beta_{H 1} H_{1}+\beta_{H 2} H_{2}+\cdots+ \\
& \beta_{C 1} C_{1}+\beta_{C 2} C_{2}+\cdots+\beta_{P P 1} P P_{1}+\beta_{P P 2} P P_{2}+\cdots+u_{L}
\end{aligned}
$$

En la ecuación 9, $L$ es el resultado de la percepción de la calificación escolar y controla todas las variables de cada categoría de estudio, donde: $N$ se refiere a variables de tipo individual, $H$ son las covariables del hogar, $C$ son aquellas características de la comunidad, $P P$ las políticas públicas y $u$ captura el error aleatorio. 


\section{RESULTADOS}

El cuadro 4 presenta las estimaciones de impacto del Programa de Becas Escolares sobre la percepción de los tutores acerca de las calificaciones generales de sus hijas e hijos durante el ciclo escolar 2014-2015.

Los modelos estimados son de dos tipos: MCO (columna 1) y probit ordenado (columna 2). Ambos modelos son estimados con base en la función de producción de los logros escolares presentada en el marco de referencia. Por ello, en los modelos se controla por cuatro tipos de regresores: las características de los estudiantes, los antecedentes de su hogar, los efectos fijos de las comunidades y, desde luego, por el shock de política pública que hace referencia al Programa de Becas Escolares y sobre el que se desea evaluar el impacto.

Los coeficientes tanto de MCO como de probit ordenado muestran que, controlada por otras características, la participación en el Programa de Becas Escolares aumenta la percepción de los tutores de mejores calificaciones por parte de sus hijos.

De acuerdo con el modelo de MCO, ser beneficiario podría generar un incremento de $24.9 \%$ en dicha percepción. Si bien, el modelo de probit ordenado resulta una mejor especificación debido a la naturaleza de categorías múltiples de la variable dependiente, la interpretación de dicho coeficiente no es directa como la del modelo de Mco, aunque éste confirma que la relación entre las dos variables es positiva y estadísticamente significativa. Para una mejor interpretación de estos resultados, se obtuvieron los efectos marginales de dicha especificación, los cuales se presentan al final de esta sección.

Por otra parte, los resultados empíricos confirman la relación de la función de producción de logros escolares establecida en el marco teórico, que sigue la metodología propuesta por Glewee y Lambert (2010) y Glewwe y Kremer (2006) para países en desarrollo. En este sentido, aparte del shock de política pública causado por la asignación de una beca escolar, el cual incide de manera positiva sobre la percepción que los tutores tienen de las calificaciones escolares de sus hijos, el indicador de resultados escolares es determinado por otros tres tipos de características: aspectos de cada estudiante, antecedentes de sus familias y las particularidades de las comunidades en las que habitan.

Específicamente se encontraron relevantes las siguientes variables del estudiante: nivel de estudio, relación con compañeros, comportamiento escolar y 
horas de estudio. ${ }^{10}$ Las madres o padres de estudiantes que tienen una buena relación con compañeros percibieron un mayor desempeño académico. El incremento fue de $25.5 \%$, aunque la relación fue no estadísticamente significativa. La percepción de buenas calificaciones aumentó de forma significativa cuando los estudiantes tienen un buen comportamiento escolar (58.2\%) y cuando dedican más tiempo a estudiar (20.0\%).

En lo que corresponde al grupo de covariables de los antecedentes del tutor, el impacto de la escolaridad va en el sentido esperado. Los tutores con menor escolaridad tienen una menor percepción de calificaciones altas. Cuando la escolaridad máxima es secundaria incompleta o primaria completa disminuyen significativamente su percepción de buenas calificaciones en 58.9 y $2.1 \%$, respectivamente. Esto podría estar relacionado con el hecho de que los padres con menor escolaridad carecen de herramientas suficientes para asistir a sus hijos con las tareas escolares, en comparación con los padres que tienen mayor escolaridad (Glewwe, 2005).

Los menores que tienen una relación de hijo del jefe o jefa del hogar reducen la percepción de altas calificaciones por parte de sus tutores (-66.1\%). Cuando la madre es la responsable de los asuntos escolares del estudiante, la percepción de buenas calificaciones aumenta 29.6\%. En cambio, el hecho de que la tutora sea mujer o el tutor en cuestión esté empleado, disminuye la percepción (60.0 y 24.6\%, respectivamente). De acuerdo con algunos estudios para países en desarrollo (Huisman et al., 2010; Huisman y Smits, 2012), lo anterior podría deberse a que, entre la población vulnerable, las madres suelen trabajar para conseguir mayores ingresos familiares más que por un signo de empoderamiento. Dicha situación podría implicar una carencia de tiempo entre las mujeres para realizar otras actividades como el cuidado escolar de los menores.

Otro atributo de los hogares son los recursos que deciden invertir en la educación de sus niñas y niños, así como en la compra de otros bienes. Los recursos destinados a la educación pueden ser de tipo económico o de tiempo de acompañamiento. Los recursos económicos están representados en nuestro modelo por el gasto en educación, en salud, en vivienda y la disponibilidad de Internet en casa como herramienta de aprendizaje. Por su parte, el tiempo de acompańamiento es medido por la frecuencia con la que los tutores revisan las tareas escolares de sus hijos.

10 Factores controlados a partir de las preguntas del cuestionario en las que la madre, el padre o el tutor expresan: 1) si hay una buena relación entre el/la menor y sus compañeros; 2) si los estudiantes mostraron problemas de comportamiento, y 3 ) el tiempo que dedican los menores a estudiar por día. 
El gasto en educación no tuvo un efecto significativo sobre la percepción de mejores calificaciones escolares, lo cual podría atribuirse a que este rubro es en promedio de MXN\$200 mensuales y representa apenas $9.4 \%$ del gasto total mensual de los hogares de la muestra. Por su parte, el gasto en salud mostró también un efecto nulo y no significativo sobre la percepción de calificaciones. Esto también podría estar relacionado con el bajo monto que invierten las familias en salud, el cual es de MXN\$118 mensuales y representa 3.5\% del gasto total mensual. A pesar de esto, el gasto en vivienda podría ser un gasto relevante en estas familias, ya que representa $13.8 \%$ del gasto total. Por esta razón, dicho gasto podría tener un impacto mayor en la reducción del ingreso familiar, este efecto que causa el gasto en vivienda podría reducir la inversión en educación y, por lo tanto, la percepción de buenas calificaciones.

Por su parte, la disponibilidad de Internet en los hogares sí mostró un efecto positivo y estadísticamente significativo sobre la percepción de los tutores de mejores calificaciones escolares de sus hijos: incrementa $26.6 \%$ dicha percepción. La frecuencia con la que los tutores revisan las tareas escolares de los estudiantes incrementa también la percepción de mejores calificaciones $19.9 \%$, aunque esta relación no es significativa.

En términos de los efectos de la comunidad, se refiere al tiempo que invierten en transportarse los niños para ir de su casa a la escuela, y que muestra una relación negativa con la percepción de los tutores de mejores calificaciones en sus hijos. Finalmente, se incluyen en las estimaciones los efectos fijos por cada alcaldía, con la finalidad de capturar las diferencias espaciales.

Cuadro 4. Estimaciones de impacto del Programa de Becas Escolares en la percepción de promedio

\begin{tabular}{lcc}
\hline Determinantes & $\begin{array}{c}\text { MCO } \\
(1)\end{array}$ & $\begin{array}{c}\text { Probit ordenado } \\
\text { (2) }\end{array}$ \\
\hline Características del estudiante & & \\
\hline Participación en Becas Escolares & $0.249^{\star \star}$ & $0.496^{\star \star}$ \\
& $(0.125)$ & $(0.238)$ \\
Nivel: secundaria & $-0.253^{\star}$ & $-0.533^{\star \star}$ \\
& $(0.142)$ & $(0.263)$ \\
Relación con compañeros & 0.255 & 0.395 \\
& $(0.181)$ & $(0.331)$
\end{tabular}


Becas Escolares y su impacto en la percepción del promedio escolar

\begin{tabular}{lcc}
\hline Determinantes & MCO & Probit ordenado \\
& $(1)$ & $(2)$ \\
\hline Comportamiento en la escuela & $0.582^{\star \star}$ & $1.147^{\star \star}$ \\
& $(0.266)$ & $(0.519)$ \\
Horas de estudio & $0.200^{\star \star}$ & $0.422^{\star \star \star}$ \\
Hijo del jefe del hogar & $(0.082)$ & $(0.157)$ \\
& $-0.661^{\star \star}$ & $-1.545^{\star \star \star}$ \\
& $(0.272)$ & $(0.575)$ \\
\hline
\end{tabular}

\begin{tabular}{|c|c|c|}
\hline Características del tutor & & \\
\hline \multirow[t]{2}{*}{ Escolaridad secundaria incompleta } & $-0.589 \star \star$ & $-1.154^{\star \star \star}$ \\
\hline & $(0.227)$ & $(0.422)$ \\
\hline \multirow[t]{2}{*}{ Escolaridad primaria completa } & -0.021 & -0.043 \\
\hline & $(0.191)$ & $(0.359)$ \\
\hline \multirow[t]{2}{*}{ La madre es quien se encarga del menor } & 0.296 & 0.607 \\
\hline & $(0.256)$ & $(0.489)$ \\
\hline \multirow[t]{2}{*}{ Género del tutor } & $-0.600^{\star}$ & $-1.173^{\star}$ \\
\hline & $(0.338)$ & $(0.646)$ \\
\hline \multirow[t]{2}{*}{ Tutor está empleado } & $-0.246^{\star}$ & $-0.484^{\star}$ \\
\hline & $(0.136)$ & $(0.257)$ \\
\hline \multirow[t]{2}{*}{ Frecuencia de ayuda en tareas } & 0.199 & 0.433 \\
\hline & $(0.180)$ & $(0.328)$ \\
\hline \multicolumn{3}{|l|}{ Características del hogar } \\
\hline \multirow[t]{2}{*}{ Teléfono en casa } & $-0.358^{\star \star}$ & $-0.766^{\star \star \star}$ \\
\hline & $(0.150)$ & $(0.287)$ \\
\hline \multirow[t]{2}{*}{ Internet en casa } & $0.266^{\star}$ & $0.521^{\star}$ \\
\hline & $(0.145)$ & $(0.267)$ \\
\hline \multirow[t]{2}{*}{ Gastos de las familias en educación } & 0.000 & 0.000 \\
\hline & $(0.000)$ & $(0.000)$ \\
\hline
\end{tabular}

Continúa 
Cuadro 4. Estimaciones de impacto del Programa de Becas Escolares en la percepción de promedio (continuación)

\begin{tabular}{lcc}
\hline Determinantes & MCO & Probit ordenado \\
$(1)$ & & \\
\hline Características del hogar & 0.000 & 0.000 \\
\hline Gastos de las familias en salud & $(0.000)$ & $(0.000)$ \\
Gastos de las familias en vivienda & $-0.000^{\star \star}$ & $-0.001^{* \star}$ \\
\hline Características de la comunidad & $(0.000)$ & $(0.000)$ \\
\hline Tiempo de desplazamiento a la escuela & & -0.002 \\
Alcaldías & -0.000 & 0.006 \\
\hline Constante & 0.003 & Sí \\
\hline R-cuadrada & Sí & \\
\hline
\end{tabular}

Notas: Significancia: ${ }^{\star} p<0.10 ;{ }^{\star \star} p<0.05 ;{ }^{\star \star \star} p<0.01$. Error estándar entre paréntesis.

Fuente: elaboración propia con datos de la Encuesta de Evaluación de Impacto del Programa de Becas Escolares de la Ciudad de México (2016).

Como se mencionó, el coeficiente de la ecuación de probit ordenado no tiene una interpretación directa, aunque puede emplearse para calcular las probabilidades de obtener determinada calificación y que fueron obtenidas mediante los efectos marginales de la variable referente al shock de política pública. El cuadro 5 presenta estos cambios marginales. Los datos revelan que los estudiantes que participan en el programa aumentan $18.1 \%$ la probabilidad de que sus tutores perciban una calificación más favorable de entre nueve y diez. Al mismo tiempo, este grupo disminuye la probabilidad de percibir calificaciones de rango intermedio y bajo (11.3 y 6.7\% menor, respectivamente). Dichas probabilidades son estadísticamente significativas para todos los niveles de la variable dependiente. 
Cuadro 5. Impacto de la participación en el Programa de Becas Escolares sobre la probabilidad de percibir diferentes calificaciones

\begin{tabular}{lccc}
\hline & Tratamiento $=1$ & Control $=0$ & Impacto \\
\hline $\mathrm{P}[\mathrm{L}=1$ o entre 7 y 8$]$ & 0.049 & 0.117 & -0.067 \\
$\mathrm{P}[\mathrm{L}=2$ o entre 8 y 9] & 0.397 & 0.511 & -0.113 \\
$\mathrm{P}[\mathrm{L}=3$ o entre 9 y 10] & 0.553 & 0.372 & 0.181 \\
\hline
\end{tabular}

Fuente: elaboración propia con datos de la Encuesta de Evaluación de Impacto del Programa de Becas Escolares de la Ciudad de México (2016).

\section{CONCLUSIONES}

Una estrategia de política pública ampliamente usada en los países en desarrollo, para fomentar la inversión en capital humano de la población con menores recursos, es la implementación de programas de transferencias monetarias para la educación. En la Ciudad de México se aplicó en 2001 el Programa de Becas Escolares con la finalidad de incentivar a niñas y niños de los hogares más vulnerables a permanecer en la escuela y concluir exitosamente sus estudios de nivel básico. El programa brinda a estudiantes de educación pública de nivel primaria y secundaria una transferencia monetaria de MXN\$800 mensuales ${ }^{11}$ y la posibilidad de participar en actividades lúdicas y culturales.

Este tipo de programas aumentan los recursos disponibles de los hogares, estimulan así la permanencia escolar. Sin embargo, podrían generar también un cambio en la percepción de los padres sobre el rendimiento escolar de sus hijas e hijos, con lo que también se modifican sus expectativas. En este sentido, la percepción es relevante, ya que está vinculada con el proceso de aprendizaje, principalmente a través de la motivación de madres y padres de familia. Dicha motivación está correlacionada con los resultados de permanencia y calificaciones escolares (Gutman y Akerman, 2008; Chiapa et al., 2012).

Este artículo constituye un aporte a la literatura científica al estimar por primera ocasión los efectos de la participación en el Programa de Becas Escolares de la Ciudad de México sobre la percepción que tienen los padres de las calificaciones escolares de sus hijos. El estudio compara, mediante un enfoque contrafactual, los resultados de estudiantes beneficiarios y no beneficiarios.

11 El monto de la beca fue de MXN\$600 en 2001, se incrementó a MXN\$688 en 2005, a MXN\$787.80 en 2009 y a MXN\$800 en 2015 (Gaceta Oficial Distrito Federal, 2017). 
Los datos empleados se obtuvieron del levantamiento de encuestas para la primera evaluación de impacto del programa, la cual permitió contar con información sobre la percepción del promedio académico para el ciclo escolar 2014-2015, tanto para el grupo de niñas y niños que recibió la beca como para el grupo que no la recibió. El diseño para el levantamiento de cuestionarios consistió en un muestreo aleatorio con estratificación por unidad territorial de la Ciudad de México.

Las estimaciones están basadas en una función de producción de desempeño escolar, medido a través de las calificaciones reportadas en la boleta. Los determinantes son de cuatro categorías: individual, familiar, de la localidad, así como shocks de política pública. Este último está representado en el modelo por la disponibilidad del programa de transferencias monetarias. La estrategia de estimación consistió en modelos de regresión lineal y modelos de probit ordenado, donde este último resulta un modelo más adecuado debido a la condición categórica de la variable dependiente.

Los hallazgos indican que, una vez que se controla por el resto de los factores, el programa de Becas Escolares genera un impacto positivo sobre la percepción de los tutores de mejores calificaciones en sus hijas e hijos. Una mejora en la percepción es importante para que los tutores se sientan motivados a que sus niñas y niños permanezcan en la escuela por más tiempo y no en otras actividades. Particularmente las estimaciones de MCO muestran un coeficiente de $24.9 \%$ en la mejora de la percepción. Los efectos marginales obtenidos a partir de la regresión de probit ordenado indican que los padres de estudiantes que reciben la beca escolar aumentan la percepción de calificaciones más altas (entre nueve y diez) 18.1\%. Al mismo tiempo, reducen la percepción de promedios de rango intermedio (entre ocho y nueve) y promedios de menor categoría (entre siete y ocho) 11.3 y $6.7 \%$, respectivamente.

El análisis de la efectividad de los programas de política pública, tal como el de Becas Escolares, resulta de gran relevancia para conocer la efectividad de las acciones implementadas, tanto en indicadores objetivo como en resultados colaterales y, con base en ello, fortalecer o en su caso reencausar dichas estrategias. Este estudio representa una línea de base del programa y abre la posibilidad para investigaciones de seguimiento que permitan la implementación de diferentes estrategias para la estimación de los efectos temporales. 


\section{BIBLIOGRAFÍA}

Behrman, J., Parker, S. y Todd, P. (2009), "Schooling impacts of conditional cash transfers on young children: evidence from Mexico", Economic Development and Cultural Change, vol. 57, núm. 3, DOI <https://doi.org/ $10.1086 / 596614>$

Brauw, A., Gilligan, D., Hoddinott, J. y Roy, Sh. (2015), "The impact of Bolsa Família on schooling", World Development, vol. 70, DoI <https:// doi.org/10.1016/j.worlddev.2015.02.001>

Chiapa, C., Garrido, J. L. y Prina, S. (2012), "The effect of social programs and exposure to professionals on the educational aspirations of the poor", Economics of Education Review, vol. 31, núm. 5, DOI <https://doi. org/10.1016/j.econedurev.2012.05.006>

Diario Oficial de la Federación (29 de marzo de 2019), Acuerdo No. 11/03/19. Primera Sección, México, Secretaría de Educación Pública.

Fiszbein, A., Schady, N., Ferreira, F. H. G., Grosh, M., Keleher, N., Olinto, P. y Skoufias, E. (2009), "Conditional cash transfers: reducing present and future poverty", World Bank Policy Research Report 47603, Washington, DC, World Bank. Recuperado de <https://openknowledge.worldbank. org/handle/10986/2597>

Garcia, S. y Hill, J. (2010), "Impact of conditional cash transfers on children's school achievement: evidence from Colombia", Journal of Development Effectiveness, vol. 2, núm. 1, DOI <10.1080/19439341003628681>

Glewwe, P. (2002), "School and skills in developing countries: education policies and socioeconomics outcomes", Journal of Economic Literature, vol. 40, núm. 2, DOI <10.1257/002205102320161258>

(2005), "The impact of health and nutrition on education in developing countries: theory, econometrics issues, and recent empirical evidence", Food and Nutrition Bulletin, vol. 26, núm. 2, DoI <https://doi. org/10.1177/15648265050262S215>

Glewwe, P. y Kremer, M. (2006), "School, teacher, and education outcomes in developing countries", Handbook of the Economics of Education, vol. 2, DOI <10.1016/S1574-0692(06)02016-2>

y Lambert, S. (2010), "Education production funtions: evidence from developing countries", en P. Peterson, E. Baker y B. McGaw, International Encyclopedia of Education, United Kingdom, Elsevier.

y Kassouf, A. (2012), "The impact of the Bolsa Escola/Familia conditional cash transfer program on enrollment, dropout rates and grade 
promotion in Brazil", Journal of Development Economics, vol. 97, núm. 2, DOI <https://doi.org/10.1016/j.jdeveco.2011.05.008>

Gutman, L. y Akerman, R. (2008), "Determinants of aspirations: wider benefits of learning research report. No. 2", Centre for Research on the Wider Benefits of Learning, Londres, Institute of Education, University of London.

Huisman, J., Rani, U. y Smits, J. (2010), "School characteristics, socioeconomic status and culture as determinant of primary school enrolment in India”, Working Paper 10-109, Nijmegen Center for Economics, Institute for Management Research Radboud University Nijmegen.

y Smits, J. (2012), "Determinants of education participation and garden differences in education in six arab countries", Working Paper 12102, Nijmegen Center for Economics, Institute for Management Research Radboud University Nijmegen.

Inter-American Development Bank (2017), How conditional cash transfers work, Washington, No. IDB-BK-166.

Órgano de Difusión del Gobierno del Distrito Federal (2015), Gaceta Oficial Distrito Federal, No. 20, Tomo I.

(2016), Gaceta Oficial Distrito Federal, No. 270, Tomo III.

(2017), Gaceta Oficial Distrito Federal, No. 255, Tomo III.

(2018), Gaceta Oficial de la Ciudad de México, No. 252, Tomo III.

Simóes, A. y Sabates, R. (2014), "The contribution of Bolsa Família to the educational achievement of economically disadvantaged children on Brazil", International Journal of Education Development, vol. 39, DOI <https://doi. org/10.1016/j.ijedudev.2014.07.012>

Stampini, M., Martinez-Cordova, S., Insfran, S. y Harris, D. (2018), "Do conditional cash transfers lead to better secondary schools? Evidence from Jamaica's PATH", World Development, vol. 101, DOI <https://doi. org/10.1016/j.worlddev.2017.08.015>

Stampini, M. y Tornarolli, L. (2012), "The growth of conditional cash transfers in Latin America and the Caribbean: did they go too far?", InterAmerican Development Bank, No. IDB-PB-185. Recuperado de <https:// publications.iadb.org/es/publicacion/growth-conditional-cash-transferslatin-america-and-caribbean-did-they-go-too-far>

Woessmann, L. y Hanushek, E. (2012), "Schooling educational achiviement, and the Latin America growth puzzle", Journal of Development Economics, vol. 99, núm. 2, DOI <https://doi.org/10.1016/j.jdeveco.2012.06.004> 